\title{
Extended Posterior Decompression and Instrumented Fusion for Spinal Tuberculosis
}

\author{
Sombat Kunakornsawat, Nattaphon Philawuth, Chaiwat Piyaskulkaew, \\ Pritsanai Pruttikul, Tinnakorn Pluemvitayaporn, Piyabuth Kittithamvongs \\ Center of Excellence in Orthopaedics, Lerdsin General Hospital, Bangkok, Thailand
}

\begin{abstract}
Study Design: Retrospective cohort.
Purpose: To evaluate clinical outcomes, including pain and neurologic status, and to evaluate radiographic outcomes of patients treated with extended posterior decompression, posterior fixation, and fusion in different vertebral segments.

Overview of Literature: The standard surgical treatment of spinal tuberculosis is radical debridement via anterior approach. However, this approach may lead to several serious complications. Meanwhile, extended posterior approach, the posterior surgical approach, involving the removal of posterior elements, ribs, and pedicles, is an alternative option that can achieve the aims of treatment in this disease and may reduce the serious complications from anterior approach.

Methods: The medical records and imaging of 50 patients admitted with spinal tuberculosis from January 2010 to June 2016 were reviewed. The Visual Analog Scale (VAS), Frankel grading scale, and kyphotic Cobb angle between the pre- and postoperative periods were used to evaluate the patients.

Results: The patients had significant improvement of VAS score in all the groups. The $T / T-L, L$, and $L-S$ group scores improved from $7.2 \pm 1.5$ to $1.7 \pm 1.2(p<0.01)$, from $8.1 \pm 1.8$ to $1.7 \pm 1.4(p<0.01)$, and from $7.9 \pm 2.2$ to $1.7 \pm 0.8(p<0.01)$, respectively, and overall, the patient scores $(n=50)$ improved from $7.8 \pm 1.4$ to $1.7 \pm 1.3(p<0.01)$. Ten patients $(20 \%)$ had Frankel grade $E$ preoperatively, which was improved to 38 patients (76\%) postoperatively. A significant improvement of the kyphotic Cobb angle was observed when compared at the preoperative, early postoperative, and final follow-up period in the T/T-L, L, and $L-S$ groups. The loss of correction angle in the $L S$ group was $7.7^{\circ} \pm 4.3^{\circ}$ at the final follow-up compared with the early postoperative correction angle at $9.1^{\circ} \pm 5.8^{\circ}$, with no statistically significant difference.

Conclusions: Extended posterior decompression, posterior instrumentation, and fusion are effective methods of surgery for treatment of spinal tuberculosis involved in the thoracic, thoracolumbar, lumbar, and lumbosacral regions.
\end{abstract}

Keywords: Thoracolumbar; Lumbosacral; Spinal tuberculosis; Transpedicular decompression

\section{Introduction}

It is difficult to diagnose and treat spinal tuberculosis. Spinal tuberculosis can cause severe neurological deficits, kyphotic deformities from vertebral collapse, and paraple- gia, thus, representing a life-threatening and disabling condition [1,2]. Treatment consists of medical and surgical modalities. Antituberculosis chemotherapy remains the mainstay of treatment. However, surgery is indicated in patients with disabling back pain or progressive neu-

Received Aug 5, 2018; Revised Feb 1, 2019; Accepted Feb 25, 2019

Corresponding author: Piyabuth Kittithamvongs

Institute of Orthopaedics, Lerdsin Hospital, 190 Silom Rd. Srivieng Bangrak, Bangkok 10500, Thailand

Tel: +66-2-3539844, Fax: +66-2-3539845, E-mail: piyabuthortho@gmail.com 
rological deficit $[3,4]$. Current surgical methods include anterior debridement with anterior fusion followed by anterior or posterior instrumentation and posterior debridement with or without posterior fixation [5-9]. Anterior approaches are quite extensive with associated morbidity [6,9-12]. Single-stage decompression and bone grafting fusion with instrumentation via the posterior approach for spinal tuberculosis have been reported by some surgeons to have good outcomes with lower complication rates $[13,14]$. However, few reports in the literature have compared the results of posterior decompression between spinal tuberculosis at the thoracic and lumbar regions.

In this study, we reported a case series of 50 patients with thoracic and lumbar tuberculosis. The objectives of this study were to evaluate the clinical outcomes, including pain and neurologic status, and to evaluate the radiographic outcomes of patients treated with extended posterior decompression, debridement, posterior fixation, and fusion in different involved vertebral segments.

\section{Materials and Methods}

The ethics committee of Lerdsin Hospital approved the study (IRB no., 0306/13/194). Written informed consents were obtained from all participants. The medical records and radiographs of 50 patients with spinal tuberculosis admitted from January 2010 to June 2016 were retrospectively reviewed. All the patients had a confirmed diagnosis by polymerase chain reaction for tuberculosis and histopathology results from surgical biopsy specimens. This study included 15 male and 35 female patients with a mean age of $51 \pm 11.4$ years. The criteria included patients with spinal tuberculosis involved in one or two adjacent segments located at the thoracic, thoracolumbar, lumbar, and lumbosacral segments who underwent extended posterior decompression, posterior fixation, and fusion. The patients were required to not have anterior abscesses extending along multiple vertebrae or not have abscesses extending along the psoas muscle. Excluded were patients with cervical tuberculosis and severe medical comorbidities. All the 50 patients were included in the study after careful review of the records. One of two spine surgeons at Lerdsin Hospital operated on each patient. Most patients presented with tuberculosis symptoms consisting of moderate fever, back pain, and weight loss. Pain was described as back pain or radicular pain, which was assessed using the Visual Analog Scale (VAS) preoperatively and at 12-month follow-up [15]. The Frankel classification was used to evaluate neurological deficits preoperatively and at 12-month follow-up [16]. The plain radiographs (anteroposterior and lateral views) were reviewed to locate the affected segments and number of affected vertebrae and to evaluate kyphotic angle of the localized spinal deformity using the Cobb angle. The affected segments were divided into three groups, including (1) thoracic/thoracolumbar segments (T/T-L group), (2) lumbar segment (L group), and (3) lumbosacral segment (L-S group). The VAS score and kyphotic Cobb angle were also used pre- and postoperatively in each group. The radiologic parameters were measured preoperatively, postoperatively, and at 12-month follow-up. All measurements were performed by one of the authors who did not perform the surgeries. Other investigations, including computed tomography, magnetic resonance imaging (MRI), hematologic examination, and histopathologic examination were also reviewed to confirm the diagnosis. All the patients received the standard tuberculous chemotherapy consisting of isoniazid $5 \mathrm{mg} /$ $\mathrm{kg} /$ day, rifampicin $10 \mathrm{mg} / \mathrm{kg} /$ day, pyrazinamide $20 \mathrm{mg} / \mathrm{kg} /$ day, and ethambutol $15 \mathrm{mg} / \mathrm{kg} / \mathrm{day}$, for at least 2 months, followed by a course of isoniazid, rifampicin, and ethambutol for 10 months.

All the patients were operated in the prone position under general anesthesia. The incision was made over the affected vertebrae. Subperiosteal dissection was performed until the posterior structures were exposed, including the spinous process, lamina, facet joints, and transverse process. Pedicle screws were placed at two levels upper and lower to the affected vertebra. Pedicle screws were also placed into the affected vertebra if the infection did not involve the pedicle. The global standard screw (Medyssey Co. Ltd., Elk Grove Village, IL, USA) pedicle screw system was used in all patients. Extended posterior decompression was performed by the removal of the rib (for thoracic region), pedicle, transverse process, and posterior elements of the affected vertebra. An additional decompression procedure was performed, which was either a hemi-laminectomy or complete laminectomy, depending on the extension of the lesion. To stabilize the spinal segment, a temporary rod was placed opposite the decompression side. Curettes were used to remove sequestra, abscesses, infected endplates, and infected discs. Contoured rods were placed, kyphotic angles were corrected, and autogenous bone grafts were placed posteriorly or posterolaterally. The patterns of the procedure were similar in the thoracic and lumbar regions. 
No anterior reconstruction with strut grafting was performed. The operative time, intraoperative blood loss, and fusion level were recorded postoperatively in all patients. To prevent postoperative infection, intravenous antibiotics (cefazolin) were administered in all the patients. For patients who were allergic to cefazolin, intravenous clindamycin was used instead. The patients were allowed to ambulate as tolerated. A thoracolumbar or lumbosacral brace was used for 3 months postoperatively. All the patients were advised to monitor their erythrocyte sedimentation rate, C-reactive protein, liver enzymes, and other adverse effects of the anti-tuberculosis drugs monthly.

An independent $t$-test was used to compare the kyphosis Cobb angle and VAS score. Pre- and postoperative VAS score were compared at 12-month follow-up. For the kyphosis Cobb angle, preoperative Cobb angle was used to compare the Cobb angle at the early postoperative and final follow-up periods (12-month follow-up). The early postoperative correction angle was defined as the difference between the preoperative and early postoperative Cobb angle. The loss of correction angle was defined as the difference between the postoperative Cobb angle at 12 months and the early postoperative Cobb angle. An independent $t$-test was used to compare the correction angle with the loss of correction angle. A $p$-value of $<0.05$ is considered to be statistically significant. The baseline characteristics were presented as mean \pm standard deviation for continuous variables and frequency (percentage) for categorical variables. Statistical analysis was performed to compare the outcomes by using the SPSS ver. 17.0 for Windows (SPSS Inc., Chicago, IL, USA).

\section{Results}

\section{Surgical condition and clinical outcomes}

Table 1 shows the baseline patient characteristics. The mean operative time and intraoperative blood loss were $149.2 \pm 47.6$ minutes and $637.0 \pm 436.5 \mathrm{~mL}$, respectively. The mean number of levels fused was $4.48 \pm 2.1$. The T/T$\mathrm{L}$ group required more segments to undergo fusion than the other groups $(5.13 \pm 1.6)$. Table 2 summarizes the outcomes after surgical treatment.

There were two patients with Frankel grade A, 13 patients with Frankel B, eight patients with Frankel grade C, 17 patients with Frankel grade D, and 10 patients with Frankel grade E preoperatively. Fifteen patients were had Frankel grades A and B (30\%) preoperatively. Two patients in the T/T-L group had Frankel grade A. The patients with Frankel grade A improved to Frankel C at 12 months postoperatively. No patients had Frankel grade A or B postoperatively at the final follow-up. For $17 \mathrm{pa}$ tients with Frankel grade D, eight patients improved to Frankel grade E postoperatively, and nine patients did not improve postoperatively. Ten patients (20\%) had Frankel grade E preoperatively, which was increased to 38 patients

Table 1. Baseline characteristics of 50 patients

\begin{tabular}{|c|c|c|c|c|}
\hline \multirow{2}{*}{ Variable } & \multicolumn{4}{|c|}{ Group } \\
\hline & Total $(n=50)$ & $\mathrm{T} / \mathrm{T}-\mathrm{L}(\mathrm{n}=22)$ & $L(n=18)$ & $L-S(n=10)$ \\
\hline Age (yr) & $51 \pm 11.4$ & $51.5 \pm 10.4$ & $54.5 \pm 13.7$ & $46.5 \pm 12.1$ \\
\hline \multicolumn{5}{|l|}{ Sex } \\
\hline Male & 15 & 5 & 6 & 4 \\
\hline Female & 35 & 17 & 12 & 6 \\
\hline Vertebral body disease & $1.96 \pm 0.53$ & $1.95 \pm 0.58$ & $1.94 \pm 0.42$ & $2.0 \pm 0.67$ \\
\hline \multicolumn{5}{|l|}{ Preoperative Frankel scale (\%) } \\
\hline A & $2(4.0)$ & $2(9.1)$ & 0 & 0 \\
\hline B & $13(26.0)$ & $6(27.3)$ & $5(27.8)$ & $2(20.0)$ \\
\hline C & $8(16.0)$ & $5(22.7)$ & $3(16.7)$ & 0 \\
\hline D & $17(34.0)$ & $6(27.3)$ & $6(33.3)$ & $5(50.0)$ \\
\hline $\mathrm{E}$ & $10(20.0)$ & $3(13.6)$ & $4(22.2)$ & $3(30.0)$ \\
\hline Preoperative Visual Analog Scale & $7.8 \pm 1.4$ & $7.2 \pm 1.5$ & $8.1 \pm 1.8$ & $7.9 \pm 2.2$ \\
\hline
\end{tabular}

Values are presented as mean \pm standard deviation, number, or number (\%). 
Table 2. Outcomes after surgical treatment

\begin{tabular}{|c|c|c|c|c|}
\hline \multirow{2}{*}{ Variable } & \multicolumn{4}{|c|}{ Group } \\
\hline & Total $(n=50)$ & $\mathrm{T} / \mathrm{T}-\mathrm{L}(\mathrm{n}=22)$ & $L(n=18)$ & $L-S(n=10)$ \\
\hline Operative time (min) & $149.2 \pm 47.6$ & $158.9 \pm 41.7$ & $143.0 \pm 52.7$ & $139.0 \pm 35.7$ \\
\hline Intraoperative blood loss (mL) & $637.0 \pm 436.5$ & $656.8 \pm 484.1$ & $647.2 \pm 423.7$ & $575.0 \pm 382.4$ \\
\hline Fusion level & $4.48 \pm 2.1$ & $5.13 \pm 1.6$ & $4.3 \pm 2.2$ & $3.3 \pm 1.4$ \\
\hline \multicolumn{5}{|l|}{ VAS } \\
\hline Preoperative VAS & $7.8 \pm 1.4$ & $7.2 \pm 1.5$ & $8.1 \pm 1.8$ & $7.9 \pm 2.2$ \\
\hline Postoperative VAS & $1.7 \pm 1.3$ & $1.7 \pm 1.2$ & $1.7 \pm 1.4$ & $1.7 \pm 0.8$ \\
\hline \multicolumn{5}{|l|}{ Kyphotic Cobb's angle $\left(^{\circ}\right)$} \\
\hline Preoperative Cobb's angle & $13.5 \pm 14.7$ & $23.5 \pm 13.5$ & $4.0 \pm 18.4$ & $-4.5 \pm 16.1$ \\
\hline Postoperative Cobb's angle & $2.0 \pm 11.9$ & $9.5 \pm 9.2$ & $-6.0 \pm 13.9$ & $-19.0 \pm 13.2$ \\
\hline Early postoperative correction angle & $11.0 \pm 12.5$ & $14.5 \pm 7.8$ & $10.0 \pm 9.1$ & $9.1 \pm 5.8$ \\
\hline Cobb's angle at 12-month follow-up & $5.0 \pm 7.4$ & $13.5 \pm 8.5$ & $0 \pm 7.1$ & $-9.0 \pm 6.4$ \\
\hline Loss of correction angle & $4.0 \pm 4.2$ & $3.5 \pm 3.8$ & $4.0 \pm 4.7$ & $7.7 \pm 4.3$ \\
\hline
\end{tabular}

Values are presented as mean \pm standard deviation. Degree in minus (-) represents lordosis.

VAS, Visual Analog Scale.

Table 3. Frankel grading according to locations

\begin{tabular}{lccccc} 
& \multicolumn{5}{c}{ Frankel scale } \\
\cline { 2 - 6 } Variable & A & B & C & D & E \\
\cline { 3 - 6 } T/TL group (n=22) & & & & & \\
\hline Preoperative score & 2 & 6 & 5 & 6 & 3 \\
\hline 12-Month follow-up & - & - & 2 & 5 & 15 \\
\hline group (n=18) & & & & & \\
\hline Preoperative score & - & 5 & 3 & 6 & 4 \\
\hline 12-Month follow-up & - & - & - & 4 & 14 \\
\hline L-S group (n=10) & & & & & \\
\hline Preoperative score & - & 2 & - & 5 & 3 \\
\hline 12-Month follow-up & - & - & 1 & - & 9 \\
\hline
\end{tabular}

(76\%) postoperatively. Table 3 summarizes the details of neurologic status according to Frankel grading.

The patients in the L group had the highest preoperative VAS scores $(8.1 \pm 1.8)$. The patients had significant improvement of VAS score in all the groups at 12 months postoperatively. The T/T-L, L, and L-S group scores improved from $7.2 \pm 1.5$ to $1.7 \pm 1.2(p<0.01)$, from $8.1 \pm 1.8$ to $1.7 \pm 1.4(p<0.01)$, and from $7.9 \pm 2.2$ to $1.7 \pm 0.8(p<0.01)$, respectively, and the overall the patient scores $(n=50)$ improved from $7.8 \pm 1.4$ to $1.7 \pm 1.3$ ( $p<0.01)$. Table 4 compares and summarizes the details of VAS score.
Table 4. Comparison between preoperative and postoperative VAS score

\begin{tabular}{|c|c|c|c|}
\hline \multirow{2}{*}{ Group } & \multicolumn{2}{|c|}{ VAS } & \multirow{2}{*}{$p$-value } \\
\hline & Preoperative & Postoperative & \\
\hline Total $(n=50)$ & $7.8 \pm 1.4$ & $1.7 \pm 1.3$ & $<0.01$ \\
\hline$T / T L(n=22)$ & $7.2 \pm 1.5$ & $1.7 \pm 1.2$ & $<0.01$ \\
\hline$L(n=18)$ & $8.1 \pm 1.8$ & $1.7 \pm 1.4$ & $<0.01$ \\
\hline$L-S(n=10)$ & $7.9 \pm 2.2$ & $1.7 \pm 0.8$ & $<0.01$ \\
\hline
\end{tabular}

Values are presented as mean \pm standard deviation. VAS, Visual Analog Scale.

\section{Radiographic outcomes}

Significant improvements of the kyphotic Cobb angles were observed when compared between the preoperative and early postoperative measurements in the $\mathrm{T} /$ $\mathrm{T}-\mathrm{L}\left(23.5^{\circ} \pm 13.5^{\circ}\right.$ to $\left.9.5^{\circ} \pm 9.2^{\circ}, p<0.01\right), \mathrm{L}\left(4.0^{\circ} \pm 18.4^{\circ}\right.$ to $\left.-6.0^{\circ} \pm 13.9^{\circ}, p<0.01\right)$, and $\mathrm{L}-\mathrm{S}$ groups $\left(-4.5^{\circ} \pm 16.1^{\circ}\right.$ to $\left.-19.0^{\circ} \pm 13.2^{\circ}, p<0.01\right)$. The patients in the $\mathrm{T} / \mathrm{T}-\mathrm{L}$ group had the highest mean degree of kyphotic correction at $14.5^{\circ} \pm 7.8^{\circ}$ in the early postoperative measurement. A significant improvement was found between the pre- and postoperative kyphotic Cobb angles at the final follow-up in the T/T-L $\left(23.5^{\circ} \pm 13.5^{\circ}\right.$ to $13.5^{\circ} \pm 8.5^{\circ}$, $p<0.01), \mathrm{L}\left(4.0^{\circ} \pm 18.4^{\circ}\right.$ to $\left.0^{\circ} \pm 7.1^{\circ}, p<0.01\right)$, and $\mathrm{L}-\mathrm{S}$ groups $\left(-4.5^{\circ} \pm 16.1^{\circ}\right.$ to $\left.-9.0^{\circ} \pm 6.4^{\circ}, p<0.01\right)$. The patients in the 


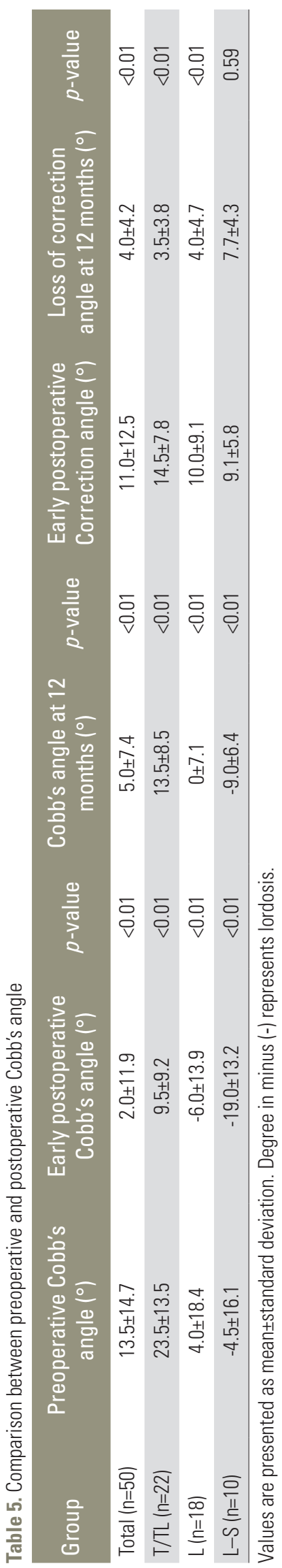

L-S group had the highest mean loss of correction at $6.5^{\circ}$ at the final follow-up. The patients in the T/T-L group showed the lowest mean loss of correction at $3.5^{\circ} \pm 3.8^{\circ}$. The loss of correction angle was $7.7^{\circ} \pm 4.3^{\circ}$ at the final follow-up compared with the early postoperative correction angle at $9.1^{\circ} \pm 5.8^{\circ}$ in the LS group, with no statistically significant difference $(p=0.59)$. Table 5 shows the details of radiologic outcomes.

\section{Case presentation}

A 48-year-old male patient with spinal tuberculosis experienced back pain for 9 months and incomplete spinal cord paralysis for 1 month (Frankel grade C). Preoperative radiography showed destructive segments located at the T8-T9 and L1-L2. The Cobb angle from the radiograph was $30^{\circ}$ at the L1-L2. MRI showed the presence of a local abscess and severe spinal cord compression. Extended posterior decompression and posterior instrumented fusion were performed at the L1-L2. Postoperative radiography showed that fixation was in good position, kyphosis had improved significantly, and the Cobb angle was $12^{\circ}$ in kyphosis. At the 12-month follow-up period, the patient's Frankel grade had recovered to E. The radiograph at 12 months of follow-up showed the union of L1-L2 vertebrae with Cobb angle at $14^{\circ}$ in kyphosis. Fig. 1 shows the radiograph and MRI.

\section{Discussion}

Spinal tuberculosis can be associated with neurological deficits due to compression of the adjacent neural structures and present with significant spinal deformity due to vertebral collapse; therefore, early management of this disease can prevent serious complications. In patients with neurologic complications, medical therapy is still the first-line of treatment; however, a combination of medical and surgical modalities yields the best results when indicated. In patients presenting with spinal deformity and spinal instability, a group who usually had a delay in initial treatment, surgical options, including debridement, fixation, and fusion, are important to provide good clinical outcomes.

Previously, the anterior approach for debridement with anterior interbody fusion described by Hodgson et al. [17] in the 1960s became popular. It allows direct access to the lesion, provides good visualization, and allows complete 

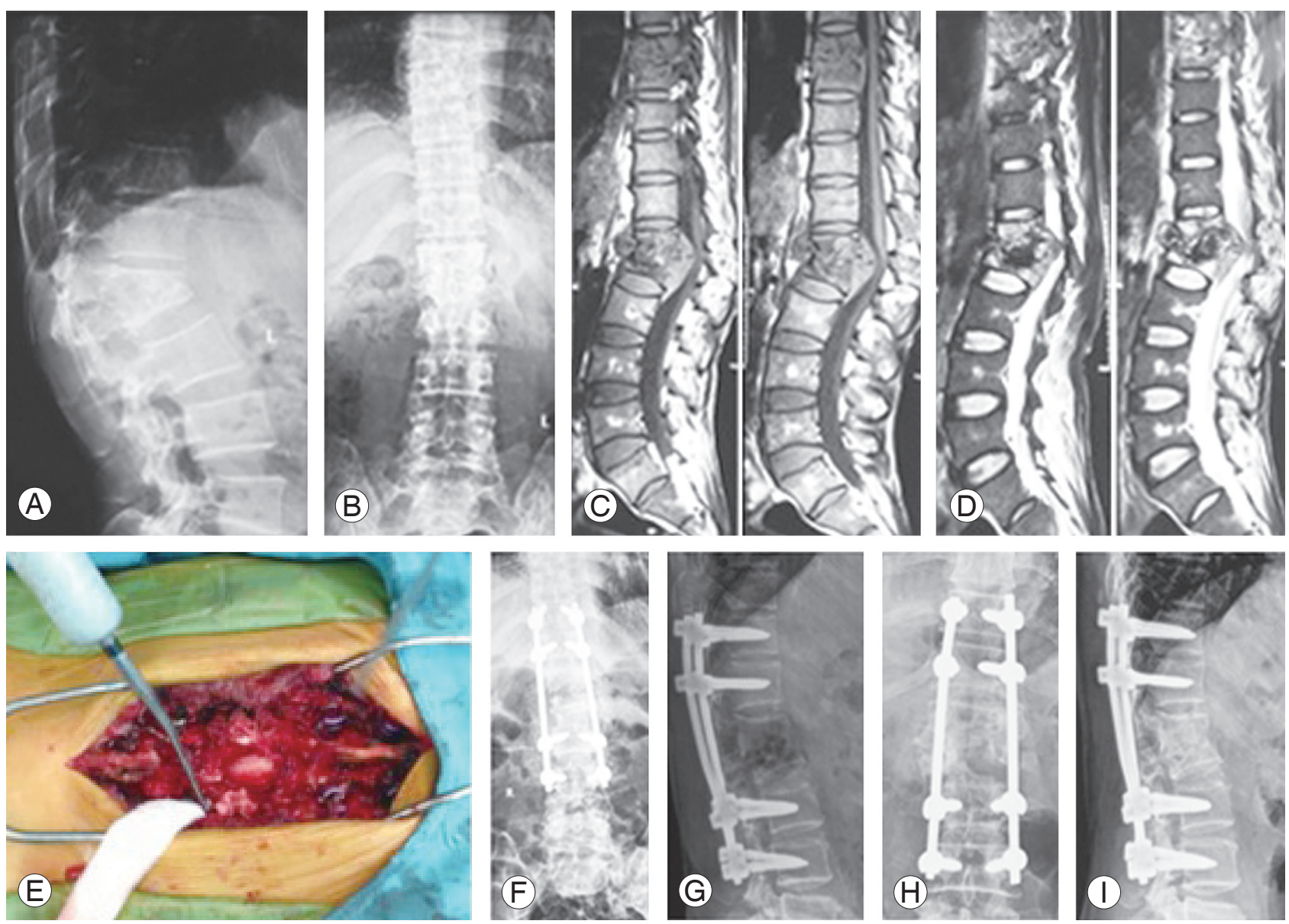

Fig. 1. Preoperative radiograph showing destructive segments at the T8-T9 and $L 1-L 2$. (A, B) The Cobb angle from the radiograph was $30^{\circ}$ at the L1-L2. (C, D) Magnetic resonance imaging showing paravertebral abscess with spinal cord compression. (E) Transpedicular decompression is performed at the L1-L2 on the right. (F, G) Early postoperative radiograph shows that fixation was in good position, kyphosis has significantly improved, and the Cobb angle is $12^{\circ}$ in kyphosis. $(\mathrm{H}, \mathrm{I})$ The radiograph at the 12 -month follow-up period shows the union of the L1-L2 vertebrae with the Cobb angle at $14^{\circ}$ in kyphosis.

spinal cord decompression. This approach has been widely regarded as the gold standard of treatment [17]. However, many surgeons have concern over its possible severe complications, including direct injury of large vessels, neurological injuries (cord, roots, nerves), late thrombophlebitis, thrombophlebitis, sympathicolysis, and various thoracic cavity morbidities, such as atelectasis, pleural effusion, and possible spread of tuberculosis in the chest [18]. In recent years, the posterior approach for debridement, instrumentation, and fusion for the thoracolumbar spinal tuberculosis is performed by many surgeons more frequently. Huang et al. [19] recently compared posterior and anterior debridement in combination with bone graft and internal fixation for lumbar and thoracic tuberculosis and found similar clinical efficacy in terms of pain control, Cobb angle, and neurological function. Luo et al. [20] studied the single-stage transpedicular approach in 37 cases of thoracic spinal tuberculosis with elderly patients and found good results for kyphotic angle without mortality in all 36 cases achieving solid fusion. Zhang et al. [21] investigated a one-stage posterior surgical approach for upper thoracic spinal tuberculosis by internal fixation, debridement, and combined interbody and posterior fusion. The results showed significant postoperative improvement in American Spinal Injury Association classification scores. The thoracic kyphotic angles were significantly decreased to $12^{\circ}-26^{\circ}$ postoperatively, and were $13^{\circ}-28^{\circ}$ at the final follow-up, without serious complications.

In our experience, extended posterior debridement with posterior instrumentation and fusion has many advantages. First, it can be performed completely in one incision and surgical position, provides short intraoperative time, and less intraoperative blood loss. Second, it avoids one-lung ventilation, causing fewer postoperative 
complications. These complications, including atelectasis, chylothorax, lung infection, and avoidance of major vascular structure injury. This procedure can also be safely performed in patients with severe pleural adhesions. Third, good exposure can be expected from this approach due to removal of the ribs, transverse process, and small joints on one side, supplying adequate operative space for exposure of the lesions. Fourth, it provides intraoperative stability by using temporary rods before decompression and debridement so that the spinal cord and spinal nerves can be protected. Posterior instrumentation and fusion are used to restore postoperative stability. If possible, a unilateral laminectomy is performed to increase the area of the posterior fusion. Fifth, an extended laminectomy can be performed posteriorly to decompress the spinal cord and nerve roots in patients with concomitant spinal stenosis. However, there are some concerns in patients with large prevertebral abscesses or destruction to multiple vertebrae because achievement of satisfactory debridement by the only-posterior approach in these patients can be difficult.

Our results showed that the kyphotic Cobb angle could be corrected in all spinal segments with only $3.5^{\circ}$ loss of correction observed in thoracolumbar segments at the final follow-up. However, progressive kyphotic deformity could be observed in the lumbosacral segments. The loss of correction angle at the lumbosacral segment was $7.7^{\circ}$ at final follow-up compared with the early-postoperative correction angle at $9.1^{\circ}$ with no statistically significant difference. These constituted $85 \%$ loss of correction at lumbosacral area after posterior fixation and fusion. This finding can be explained by the mobility of the lumbosacral segment without the anterior column support and inadequate sacral fixation, usually inserting S1 pedicle screws only. In these cases, an interbody fusion may be added to support the anterior spinal column for spinal tuberculosis involving the lumbosacral segment $[21,22]$ or extended sacral fixation such as S2AI screw [23] may be performed to prevent progressive kyphotic deformity. This study has several limitations. First, we did not measure the angle before the 12-month postoperative followup routinely. Therefore, we could not demonstrate the exact time of correction loss. Second, because this study was a retrospective study obtained from a small number of the patients with relatively short follow-up period, further prospective study with a larger number of patients and longer follow-up period will be required.

\section{Conclusions}

In sum, our study demonstrates that an effective method for the treatment of spinal tuberculosis involving the thoracic, thoracolumbar, lumbar, and lumbosacral regions is extended posterior decompression, posterior instrumentation, and fusion for spinal tuberculosis. This procedure stabilizes the vertebral segments intraoperatively, preventing spinal cord injury and avoids pulmonary and vascular morbidities. It can provide good pain score reduction, improve neurologic status, and maintain good postoperative sagittal alignment. For the lumbosacral region, an additional interbody fusion or an extended sacral fixation may be performed to prevent progressive kyphotic deformity.

\section{Conflict of Interest}

No potential conflict of interest relevant to this article was reported.

\section{References}

1. Muangchan C, Nilganuwong S. The study of clinical manifestation of osteoarticular tuberculosis in Siriraj Hospital, Thailand. J Med Assoc Thai 2009;92 Suppl 2:S101-9.

2. Tuli SM, Srivastava TP, Varma BP, Sinha GP. Tuberculosis of spine. Acta Orthop Scand 1967;38:445-58.

3. Jain AK, Kumar J. Tuberculosis of spine: neurological deficit. Eur Spine J 2013;22 Suppl 4:624-33.

4. Nussbaum ES, Rockswold GL, Bergman TA, Erickson DL, Seljeskog EL. Spinal tuberculosis: a diagnostic and management challenge. J Neurosurg 1995;83:243-7.

5. Lee BH, Park JO, Kim HS, Lee HM, Cho BW, Moon SH. Transpedicular curettage and drainage versus combined anterior and posterior surgery in infectious spondylodiscitis. Indian J Orthop 2014;48:7480.

6. Jayaswal A, Upendra B, Ahmed A, Chowdhury B, Kumar A. Video-assisted thoracoscopic anterior surgery for tuberculous spondylitis. Clin Orthop Relat Res 2007;460:100-7.

7. Lee SH, Sung JK, Park YM. Single-stage transpedicular decompression and posterior instrumentation in treatment of thoracic and thoracolumbar spinal tuberculosis: a retrospective case series. J Spinal Disord 
Tech 2006;19:595-602.

8. El-Sharkawi MM, Said GZ. Instrumented circumferential fusion for tuberculosis of the dorso-lumbar spine: a single or double stage procedure? Int Orthop 2012;36:315-24.

9. Sundararaj GD. Simultaneous anterior decompression and posterior instrumentation of the tuberculous spine using an anterolateral extrapleural approach. J Bone Joint Surg Br 2009;91:702.

10. Jain AK. Treatment of tuberculosis of the spine with neurologic complications. Clin Orthop Relat Res 2002;398:75-84.

11. Okada Y, Miyamoto H, Uno K, Sumi M. Clinical and radiological outcome of surgery for pyogenic and tuberculous spondylitis: comparisons of surgical techniques and disease types. J Neurosurg Spine 2009;11:620-7.

12. Lu G, Wang B, Li J, Liu W, Cheng I. Anterior debridement and reconstruction via thoracoscopy-assisted mini-open approach for the treatment of thoracic spinal tuberculosis: minimum 5-year follow-up. Eur Spine J 2012;21:463-9.

13. He B, Hu Z, Hao J, Liu B. Posterior transpedicular debridement, decompression and instrumentation for thoracic tuberculosis in patients over the age of 60. Arch Orthop Trauma Surg 2012;132:1407-14.

14. Zhang HQ, Li JS, Zhao SS, et al. Surgical management for thoracic spinal tuberculosis in the elderly: posterior only versus combined posterior and anterior approaches. Arch Orthop Trauma Surg 2012;132:1717-23.

15. Briggs M, Closs JS. A descriptive study of the use of visual analogue scales and verbal rating scales for the assessment of postoperative pain in orthopedic patients. J Pain Symptom Manage 1999;18:438-46.

16. Frankel HL, Hancock DO, Hyslop G, et al. The value of postural reduction in the initial management of closed injuries of the spine with paraplegia and tetraplegia: I. Paraplegia 1969;7:179-92.

17. Hodgson AR, Stock FE, Fang HS, Ong GB. Anterior spinal fusion: the operative approach and pathological findings in 412 patients with Pott's disease of the spine. Br J Surg 1960;48:172-8.

18. Moon MS, Kim SS, Moon YW, Moon H, Kim SS. Surgery-related complications and sequelae in management of tuberculosis of spine. Asian Spine J 2014;8:435-45.

19. Huang Y, Lin J, Chen X, Lin J, Lin Y, Zhang H. A posterior versus anterior debridement in combination with bone graft and internal fixation for lumbar and thoracic tuberculosis. J Orthop Surg Res 2017;12:150.

20. Luo C, Wang X, Wu P, Ge L, Zhang H, Hu J. Singlestage transpedicular decompression, debridement, posterior instrumentation, and fusion for thoracic tuberculosis with kyphosis and spinal cord compression in aged individuals. Spine J 2016;16:154-62.

21. Zhang H, Sheng B, Tang M, et al. One-stage surgical treatment for upper thoracic spinal tuberculosis by internal fixation, debridement, and combined interbody and posterior fusion via posterior-only approach. Eur Spine J 2013;22:616-23.

22. Sudprasert W, Choovongkomol K, Piyapromdee U, Leownorasate M. Impact on neurological recovery of transforaminal debridement and interbody fusion versus transpedicular decompression in combination with pedicle screw instrumentation for treating thoracic and lumbar spinal tuberculosis. Asian Spine J 2016;10:543-52.

23. O'Brien JR, Yu W, Kaufman BE, et al. Biomechanical evaluation of S2 alar-iliac screws: effect of length and quad-cortical purchase as compared with iliac fixation. Spine (Phila Pa 1976) 2013;38:E1250-5. 